\title{
The Classification of Leprosy
}

G. A. Ryrie.

Several attempts have been made recently to formulate modifications in or changes of the present classification of leprosy adopted by the Manila Conference in 1931. It is I think generally admitted that the present classification leaves something to be desired. The present article is an effort to discuss (a) What is fundamentally wrong with the present classification? and (b) What the essentials of a new classification should be.

What is wrong with the present classification? To begin with no progress can be macle in this matter unless it is frankly admitted that at the Manila Conference the significance and extent of the tuberculoid phases of leprosy were not fully appreciated. There is a good deal of internal evidence of this in the Report of the Leonard Wood Memorial Conference on Leprosy. For instance on page 457 it states that " in many cases that would ordinarily be described as cutaneous leprosy a history of primary nerve disturbance can be obtained and very often careful examination may reveal some degree of sensory disturbance and of nerve thickening ". Here there is obvious confusion between cutaneous and tuberculoid leprosy. Further on, the Report states "it is deemed desirable to class all cases with leprotic lesions of the skin as cutaneous ", whereas it is now clearly realised that skin tuberculoid lesions are associated with the neural form. The report discusses lepra reaction but has no recognition of acute tuberculoid leprosy : it seems a fair assumption that these two completely opposite phases of the disease were lumped together as a single entity. Whether this is unfair to the Conference or not the ordinary person reading the Report felt that all leprotic activity affecting the skin was cutaneous and that neural leprosy consisted of leprotic infiltration of the peripheral nerves and its direct atrophic consequences.

Possibly Japanese workers were clearer in the matter in dividing leprosy into maculo-anaesthetic and nodular forms. This however does not seem a very good classification. Only a minority of tuberculoid lesions are macules in the dermatological sense of the word, and anaesthesia is by no means the sole preserve of tuberculoid leprosy. Glove or stocking anaesthesia for instance is extremely common in advanced cutaneous (or nodular) cases.

Again " nodular" is a poor description of cutaneous 
leprosy. Only a small percentage of cutaneous cases are actually nodular and nodules of a tuberculoid character may occur in maculo-anaesthetic cases.

Returning to the Manila classification we find that as tuberculoid skin lesions are associated with neural leprosy they have been rather awkwardly tucked in under the inadequate $\mathrm{N}$ blanket. It is obvious, however, from the literature that some workers are still calling these lesions cutaneous because they are skin granulomata-which leads to considerable confusion.

What happens, however, if the tuberculoid lesion is considered as neural? A patient has atrophy of the hands and feet from extensive nerve involvement. He has no skin lesions. He is an N3 case.

Another patient has most of his body surface covered with tuberculoid lesions. He has only slight clinical nerve involvement. He is equally an N3 case. The symbol $\mathrm{N}$ therefore loses all its value in giving a mental picture of the patient's condition.

An attempt to solve the difficulty may be made by introducing the symbol N.M. (neuro-macular) as a sub-division of $\mathrm{N}$. This means the automatic addition of a third class. Again the word neuromacular is obviously much more suitable for describing areas of disturbed sensation and pigmentation associated with and secondary to pure nerve leprosy.

Again the symbol $\mathrm{T}$ (tuberculoid) has been suggested. To avoid confusion, however, the symbol $\mathrm{C}$ for cutaneous leprosy would have to be altered and the whole classification thereby disrupted.

The descriptions neural and cutaneous therefore are misleading and inadequate as applied to the two main divisions of leprosy.

The second fault in the present classification is that it gives no indication of the severity of the conditions. It is an area classification, quantitative not qualitative. N2 if applied to a tuberculoid case may indicate flaring major leprides or a few flat insipid macules which arouse no clinical enthusiasm. Similarly C2 may mean rapidly sprearing leprosy with erythema and general debility or just an inert area of bacillary deposit.

A third objection which is partly inherent in the first is that the classification is on a fundamentally wrong basis. To graduate into one of the two main leprosy groups the classification imposes a tissue qualification, cutaneous or neural. In actual fact, however, it does not matter a scrap 
which tissue 1s affected; what does matter is the fundamentally different character, course, and outlook of these two processes. Atrophic lesions are frequently found in advanced cutaneous cases. Tuberculoid lesions of the skin may not be cutaneous in the classification sense, but cutaneous they are nevertheless. The important point is not the location of the lesion but the recognition of two very different processes.

These clefects are cited in the hope of clarifying one's consideration of what a classification should be. A good classification should have four features:-

(i) It should indicate on a more general basis that leprosy is divided into two main groups.

(ii) It should retain as much as possible of the Manila Conference classification with which we are familiar whose symbols $\mathrm{N}$ and $\mathrm{C}$ are useful and easy tissue indicators.

(iii) It should retain a leprosy area classification. The existing 1, 2 and 3 describing slight, medium and extensive involvement seems quite suitable.

(iv) It should indicate in some way the degree of activity or the severity of the lesion.

1. We have seen that the tissue criterion is unsatisfactory in demarcating the two main leprosy groups and that it is further no indication of the essential difference between them. We have leprosy of bacillary attack (cutaneous) and we have leprosy of bodily defence (neural). The statement like any other generali ation is I admit open to detailed criticism.

But in general the essential difference is that in "cutaneous" leprosy the patient is a Minus-variant as regards resistance; in " neural " leprosy the patient is a Plus-variant as regards resistance. That and not the tissue involved is the point of practical importance.

Let us take these types as $\mathrm{M}$ and $\mathrm{P}$. $\mathrm{M}$ represents minus-variants as regards resistance (cutaneous, nodular, malignant, etc. etc.); $\mathrm{P}$ represents plus-variants as regards resistance (neurals, neuro-maculars, maculo-anaesthetics, leprides, etc. etc.).

2. To these can be attached the tissue indicators of the Manila classification. The tuberculoid-cutaneous confusion now disappears. PC2 would indicate tuberculoid lesions 
over a moderate area. MC2 would inclicate a similar area of " cutaneous" leprosy. The symbol C thus returns to its legitimate use as describing a skin lesion. The other permutations are-olovious. It is a fair criticism that some of the possible combinations of this system are highly improbable. MN1 for instance would indicate early nerve leprosy of a malignant type-which does sound a trifle hypothetical. Against this, however, we must put the gain of using cutaneous for all skin leprosy.

3. The area indicators of the Manila Classification are retained.

4. A wide variety of terms are used at present to indicate degrees of lesion activity, especially in tuberculoid cases. For clinical purposes it would be considerably better if we could employ the same terms in both the main groups when describing severity. The activity of both types could be described as acute, subacute and chronic.

'Thus PC1 chronic would indicate a strictly localised area of simple and minor tuberculoid character. PC3 acute would indicate a generalised attack of acute tuberculoid leprosy. Similarly MC3 acute would represent cutaneous lepra fever or "reaction". MC2 subacute would indicate cutaneous leprosy over a moderate area but active and spreading.

This not only has the advantage of describing lepra reaction and acute tuberculoid leprosy but also localised forms of both these conditions.

Two difficult types occur under my classification. The first is the early lesion before one knows whether the lesion will become (a) abortive, (b) tuberculoid, (c) malignant cutaneous. The second is the transitional type where the patient is changing over from tuberculoid to cutaneousfrom being a resistance plus-variant to a resistance minusvariant. There are cases in which it appears that a focus or reservoir of malignant cutaneous leprosy in one part of the body is responsible for the appearance of tuberculoid lesions in other parts. In a case seen recently there were cutaneous lesions (lepromas) on the face and tuberculoid lesions on the trunk (both confirmed by section). Patient gave a history of both lesions being of equal duration. In such cases the $\mathrm{P}$ or $\mathrm{M}$ indicating type would simply be left out, the omission indicating the inability of the examiner to place the patient in either group.

Some such system as I have described is not merely 
necessary for efficient classification of cases. Certain forms of tuberculoid lesion will shew temporary retrogression as a result of almost every form of metabolic disturbance. For instance by vaccinating certain selected tuberculoid cases one might be able to shew that vaccination appears to be an excellent treatment for leprosy. By vaccinating another group of cutaneous cases on the reaction threshold it might equally well be shewn that vaccination is a very dangerous procedure in leprosy.

Take the lepratherapy literature of the last five yearsits discordancy is amazing. It is as if three different observers described the same man as (a) two and a half feet high and thin, (b) ten feet high and fat, (c) non-existent. Methylene blue is an excellent drug, and it is also worse than useless. Solganol is a helpful auxiliary, also it just damages the patient. Treatment is good in early cases, treatment is bad in early cases. Hydnocarpus oil and esters are of no value at all, they do a slight amount of good, they produce excellent results. While there are a number of causes for this, the most important element is the lack of clear means of describing the type of case experimented on.

To give a personal example. A number of years ago I experimented with the use of trypan blue in leprosy. I naturally selected cases with well defined isolated lesions which would be photographed and observed daily (i.e. tuberculoids) and quite honestly regarded them as cutaneous. The experiment coincided with a wave of disappointment over esters treatment and the psychological effect of something new was enormously enhanced by the selective staining of the lesions. This combined with the metabolic disturbance engendered by the treatment produced excellent results-for a time. In my report I described the lesions as cutaneous. Had I described them as a group of somewhat unstable subacute tuberculoids or had there been a classification which could have conveyed this, it would have saved a great deal of time and trouble for other workers.

Some such classification as I have outlined is therefore not only necessary for clarity but is an essential for coordinated progress in therapy. 